\title{
HIGHLIGHTS
}

LYME ARTHRITIS

\section{Direct and indirect actions of Borrelia burgdorferi}

Lyme disease-an infectious disease caused by the tick-borne bacteria Borrelia burgdorferi-presents with a characteristic circular skin rash and, if left untreated, leads to arthritis and other, often severe, chronic complications. Studies have shown that B. burgdorferi induces a predominantly type $1 \mathrm{~T}$ helperlike immune response involving the expression of cytokines and chemokines, in particular those associated with the activation of monocytes/macrophages, in skin lesions and synovial fluid of patients with Lyme disease. How does this spirochete trigger this chemokine response? A paper in Arthritis Research \& Therapy shows that B. burgdorferi acts both directly and indirectly to stimulate monocytes/macrophages to secrete chemokines and thereby bridges innate and adaptive immune responses in this disease.

Using frozen samples obtained from patients with Lyme disease and normal controls, the researchers analyzed the chemokine expression profiles of peripheral blood mononuclear cells (PBMCs) after stimulation with either B. burgdorferi or interferon (IFN)- $\gamma$, or both. Unstimulated PBMCs from patients with Lyme disease secreted basal levels of CC-chemokine ligand (CCL) 2, CCL4, CXC-chemokine ligand (CXCL) 9 and CXCL10; levels of these chemokines increased on stimulation

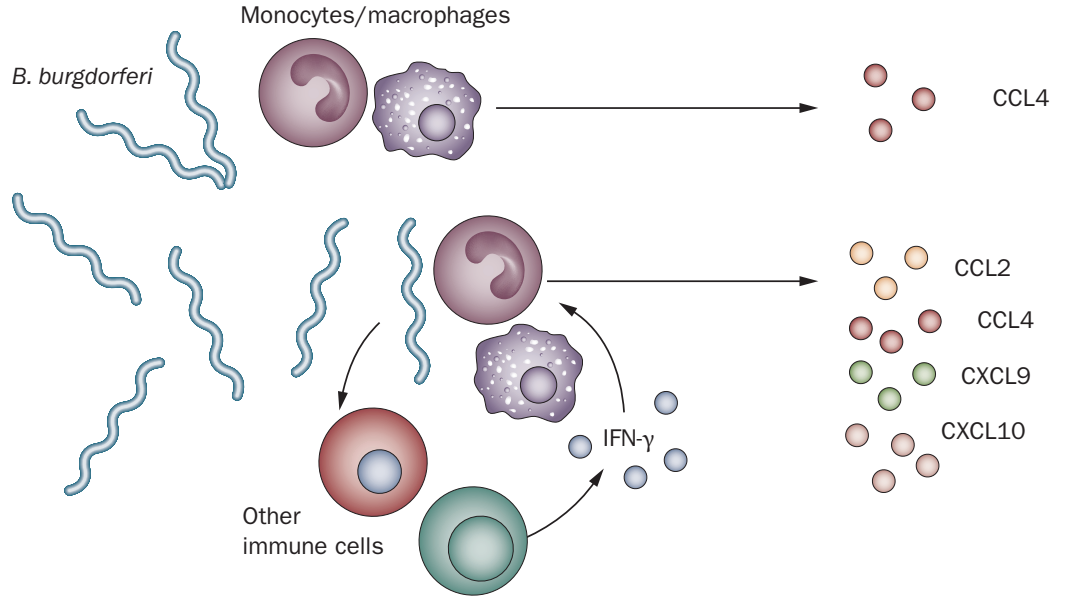

with B. burgdorferi. CD14 $4^{+}$monocytes/ macrophages, fractionated from the total PBMCs, were tested next: B. burgdorferi stimulation led to increased secretion of CCL4, but not CCL2, CXCL9 or CXCL10. CCL2 production by fractionated CD $14^{+}$ cells required both $B$. burgdorferi and IFN- $\gamma$. Stimulation with IFN- $\gamma$ alone led to production of CXCL9 and CXCL10, the levels of which were not increased by the addition of $B$. burgdorferi.

The authors conclude that $B$. burgdorferi directly stimulates $\mathrm{CD} 14^{+}$cells to produce CCL4, but that this spirochete acts indirectly, via other cell types within the PBMC population, to induce the production of IFN- $\gamma$ and thereby the secretion of additional chemokines by other innate immune cells that are key to the pathogenesis of Lyme disease.

Prof. Robert T. Schoen from Yale University School of Medicine commented that, "The complexity of the host-bacteria interaction in Lyme disease is gradually being elucidated. Shin and colleagues demonstrate that $B$. burgdorferi induces both innate and adaptive chemokine responses. The next step may be to try to evaluate how such host responses evolve over the course of the infection."

\section{Jenny Buckland}

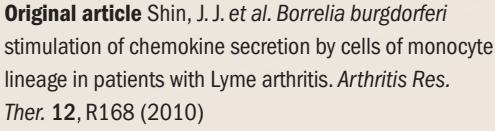

